\title{
Norois
}

Environnement, aménagement, société

$196 \mid 2005 / 3$

Géographie du littoral : approche géomatique

\section{Analyse comparative de la propriété foncière}

A partir des matrices cadastrales dans deux communes insulaires (Île-deBatz et Île-de-Bréhat)

Comparative analysis of real estate based on land registers on Bréhat and Batz inlands

\section{Clotilde Buhot}

\section{(2) OpenEdition}

\section{Journals}

Édition électronique

URL : http://journals.openedition.org/norois/427

DOI : $10.4000 /$ norois. 427

ISBN : 978-2-7535-1544-4

ISSN : $1760-8546$

Éditeur

Presses universitaires de Rennes

\section{Édition imprimée}

Date de publication : 1 septembre 2005

Pagination : 81-90

ISBN : $978-2-7535-0218-5$

ISSN : 0029-182X

Référence électronique

Clotilde Buhot, «Analyse comparative de la propriété foncière », Norois [En ligne], 196 | 2005/3, mis en ligne le 04 août 2008, consulté le 20 avril 2019. URL : http://journals.openedition.org/norois/427 ;

DOI : $10.4000 /$ norois.427

Ce document a été généré automatiquement le 20 avril 2019

(c) Tous droits réservés 


\section{Analyse comparative de la propriété foncière}

A partir des matrices cadastrales dans deux communes insulaires (Île-deBatz et île-de-Bréhat)

Comparative analysis of real estate based on land registers on Bréhat and Batz

inlands

Clotilde Buhot

\section{NOTE DE L'ÉDITEUR}

Cet article a été reçu le 4 avril 2005 et définitivement accepté le 6 octobre 2005.

1 Bien que l'espace soit en partie organisé à partir de la structure de la propriété foncière, celle-ci demeure quasiment invisible à l'œil nu; seules la forme et la disposition du parcellaire peuvent constituer des indicateurs partiels (Chaussende, 1994). Relevant en grande partie du domaine fiscal, l'identification des propriétaires est longtemps restée discrète et méconnue. Or en accordant des droits d'usages privilégiés, la législation fait des propriétaires des acteurs actifs de l'aménagement et de l'organisation de l'espace. L'étude de la propriété apparaît donc comme un préalable à la bonne compréhension de la dialectique usages du sol/organisation de l'espace.

Deux espaces insulaires sont analysés dans cet article: Bréhat et Batz. Ces deux îles présentent des contextes géographique et démographique comparables : localisées toutes deux en Manche (fig. 1) et dotées d'une superficie et d'une population voisines (318 ha et 422 habitants à Bréhat et 357 ha et 575 habitants à Batz). La principale différence vient de la nature de l'activité dominante : alors qu'à Bréhat le tourisme est omniprésent (ce qui s'exprime ici notamment par une proportion de résidences secondaires supérieure à $75 \%$ ), Batz a réussi à conserver un secteur primaire dynamique, l'agriculture maraîchère employant plus de $40 \%$ de la population active. 
Figure 1 : Localisation de Bréhat et Batz Situation of Bréhat and Batz islands

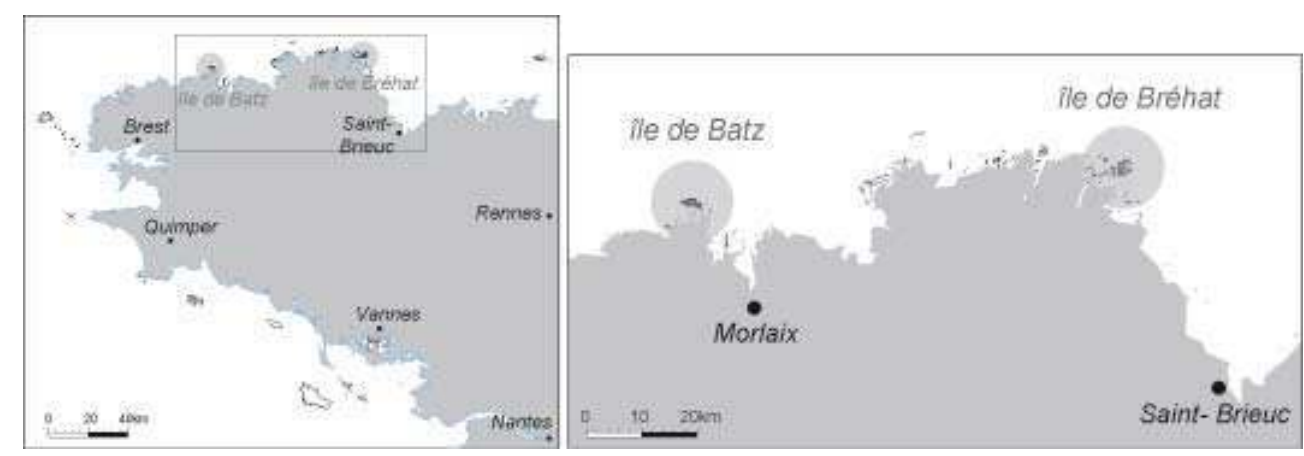

3 Une analyse de la propriété dans ce contexte permet, au-delà des indicateurs statistiques traditionnels, de mettre chaque type de propriétaire en relation avec la fraction de l'espace qu'il occupe réellement. Nous cherchons donc à déterminer comment le foncier traduit les différences existant entre ces deux îles, et quelles en sont les conséquences en matière d'organisation de l'espace. Par exemple, une concentration ou une dispersion des terres appartenant aux agriculteurs n'aura pas les mêmes conséquences sur l'espace îlien. On peut étendre ce questionnement au cas des résidents secondaires.

4 L'analyse s'effectue à l'aide d'un Système d'Information Géographique (SIG) qui permet d'allier matrices cadastrales (données statistiques) et cartographie du parcellaire; la précision de cette méthode autorise une approche multi scalaire allant de la parcelle à l'île. Les informations obtenues concernent les types de propriétaires (personnes physiques ou morales), le poids respectif de chacun d'entre eux dans la possession du sol et du bâti et leur répartition spatiale.

\section{Les matrices cadastrales : une source de données statistiques particulièrement riche}

5 En France près de 90 millions de parcelles sont cadastrées (Comby et Renard, 1996), c'està-dire représentées cartographiquement et référencées à l'aide d'un identifiant unique. Celui-ci est repris au sein d'un tableau détaillé (matrice cadastrale) qui apporte des informations concernant les parcelles qu'il représente. S'il est vrai que la mise à jour des matrices cadastrales prend parfois du retard par rapport à la propriété foncière effective, ce type de documents donne toutefois une image relativement exacte et fiable et, bien que non opposables, les informations contenues sont suffisamment pertinentes. La marge d'erreur est acceptable puisque réduite, elle ne remet ainsi pas en cause l'efficacité de la méthode.

6 Classés par ordre alphabétique, propriétaires sociétaires et collectifs d'abord, puis propriétaires individuels, ces documents constituent l'état civil de la propriété en France. Uniquement consultables sous forme de microfiches en mairie ${ }^{1}$, leur emploi implique une collecte manuelle fastidieuse, expliquant la rareté des documents de synthèse. À ce propos, J. Daligaux (1996) précise que « les chercheurs ont été unanimes pour dénoncer ce travail de bénédictin, dans lequel la lourdeur des recherches s'ajoute aux problèmes méthodologiques ». 
7 Dans le cadre de cette comparaison, qui s'inscrit par ailleurs dans notre travail de thèse portant sur les impacts du marché du logement dans les îles du Ponant, nous avons ainsi dépouillé environ 3000 relevés de propriété (correspondant à autant de propriétaires) équivalant à près de 9000 parcelles, constituant notre base de données.

Chaque relevé de propriété comprend deux types d'informations :

- le premier est d'ordre qualitatif :

- l'état civil du (ou des) propriétaire(s) lorsque celui-ci est une personne physique : nom, prénom, date et lieu de naissance, lieu de résidence principale ;

- le nom de la société ou de la collectivité lorsque le propriétaire est une personne morale ;

- les informations relatives au bien : nature de la parcelle (champs, marais, etc) ou du bâti (maison, commerce, garage, etc.) ;

- le second est d'ordre quantitatif :

- la superficie de chaque parcelle.

Enfin, à chaque parcelle sont attribués une section et un numéro de plan, constituant la référence cadastrale. Celle-ci est non seulement unique dans une commune (puisque le numéro de plan varie d'une parcelle à l'autre) mais également fondamentale puisqu'elle nous permet d'établir le lien entre notre base de données et le cadastre dont une représentation cartographique existe (fig. 2).

Figure 2 : La référence cadastrale comme lien entre cadastre et relevé de propriété The cadastral reference as a link between cadastre and register

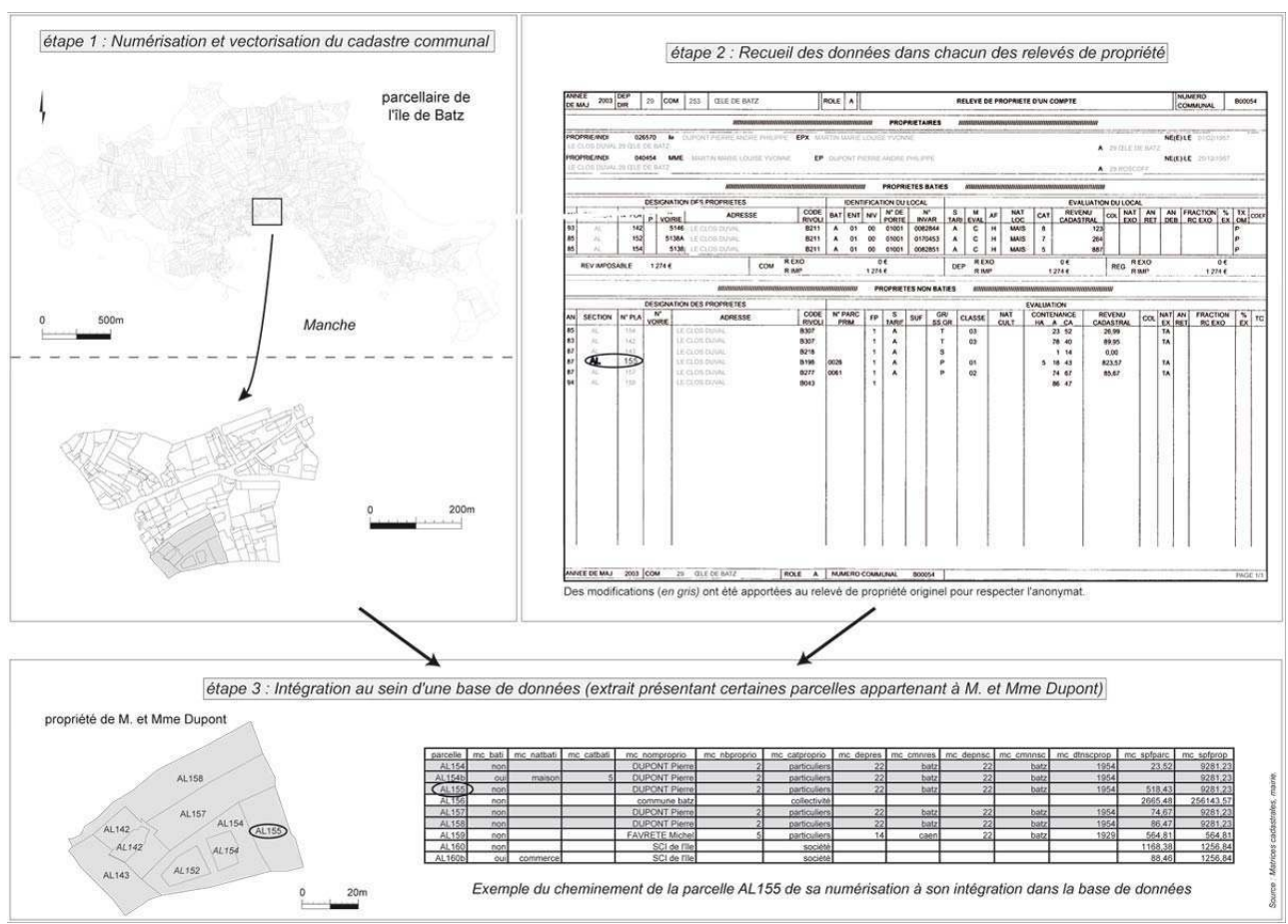

Mais paradoxalement, le cadastre est un document dont l'extrême précision nuit souvent à une réelle vision d'ensemble du parcellaire communal. En effet, la simple lecture du découpage de l'espace communal, possible uniquement une fois le travail de numérisation réalisé, nous apporte déjà des éléments de comparaison entre les deux îles, la forme et la disposition du parcellaire présentant des différences notables (fig. 3). 
Figure 3 : Répartition des propriétaires fonciers dans les îles de Bréhat et Batz (2002) Geographical distribution of land owners on Bréhat and Batz islands (2002)

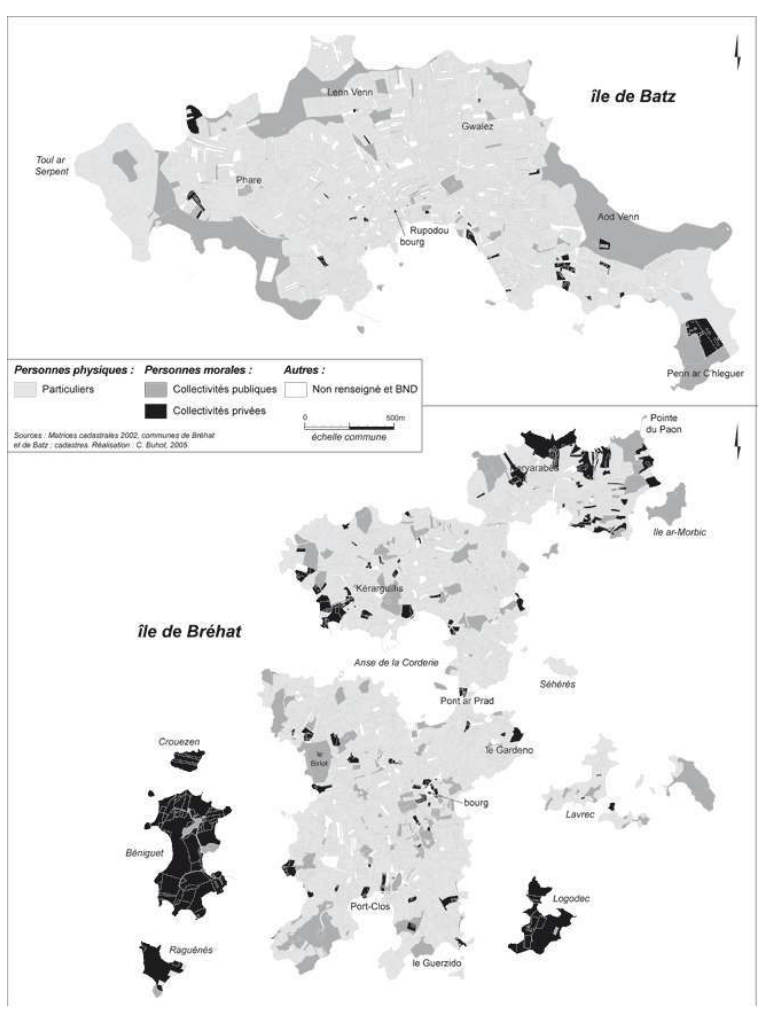

11 L'unité de base étant la parcelle, nous avons dû en effet nous appuyer sur les plans cadastraux au 1/2 000. Les cadastres de Bréhat et Batz étant disponibles uniquement sous support papier en 2002, nous avons procédé à leur numérisation puis à leur vectorisation. Nous avons donc représenté chaque parcelle par un polygone géoréférencé, lequel est relié à la base par sa section et son numéro de plan et permet de faire le lien avec la base de données (fig. 2).

\section{La prééminence des personnes physiques dans la détention du sol}

En premier lieu, il convient de relever le poids prépondérant des personnes physiques - ou particuliers - dans la possession du sol (tab. 1).

Tableau 1 : Synthèse de la propriété foncière sur Bréhat et Batz (surfaces détenues en \%) (Source : Matrices cadastrales, 2002).

Synthesis of real estate on Bréhat and Batz islands (land owned in \%)

\begin{tabular}{|l|l|l|}
\hline & Bréhat & Batz \\
\hline \hline Personnes physiques & 69,9 & 73,0 \\
\hline dont : & & \\
\hline Résidents permanents & 10,1 & 44,9 \\
\hline
\end{tabular}




\begin{tabular}{|l|l|l|}
\hline Résidents secondaires & 59,8 & 28,1 \\
\hline \hline Personnes morales & 29,8 & 26,2 \\
\hline dont : & & \\
\hline P.M. de droit public & 14,3 & 15,5 \\
\hline P.M. de droit privé & 24,3 & 1,9 \\
\hline Biens non délimités & 0,3 & 0,8 \\
\hline
\end{tabular}

13 Si le poids des personnes morales ${ }^{3}$ varie fortement, c'est que dans cette catégorie sont regroupés pêle-mêle les communes, l'État, les associations, les sociétés, les départements, etc. Ainsi, la présence du Conservatoire du Littoral, propriétaire de près de 6,6 ha à Batz, renforce le poids des personnes morales de droit public alors que la multitude de sociétés (et notamment de $\mathrm{SCI}^{4}$ ) accentue celui des personnes morales de droit privé sur Bréhat. On assiste d'ailleurs depuis quelques années à la montée en puissance des SCI dans les acquisitions de logements et de terrains, notamment sur les îles où les prix sont les plus élevés (Buhot, 2004). Quant aux biens non délimités (BND), ils désignent des parcelles où il n'a pas été procédé à un partage (entre héritiers par exemple ${ }^{5}$ ) et ne concernent, dans la grande majorité des cas, que des terrains nus et non constructibles; d'autant plus lorsqu'ils n'ont qu'une très faible valeur marchande.

En observant la figure 3, plusieurs niveaux de lecture se dégagent : si les différents types de propriétaires semblent a priori dispersés au sein de l'espace insulaire, une lecture plus attentive permet de distinguer des concentrations ponctuelles. À Batz, la localisation littorale des biens appartenant aux collectivités publiques est pour l'essentiel à mettre à l'actif de la commune; propriétaire historique des dunes (comme à Aod Venn - côte est), dont l'utilisation collective pour le séchage du goémon ou la pratique de la vaine pâture remonte au XIX ${ }^{e}$ siècle (Brigand et al., 1986); à laquelle s'ajoute l'action du Conservatoire du littoral, propriétaire de la pointe de Penn ar C’hleguer. Les terrains détenus par la commune de Bréhat sont beaucoup plus morcelés mais représentent tout de même $14 \%$ de la superficie communale (contre $22 \%$ pour celle de Batz). Les collectivités privées, dont le poids est restreint sur l'île finistérienne avec seulement quelques sociétés détentrices de propriétés (situées principalement aux alentours du bourg), sont beaucoup plus nombreuses sur le territoire bréhatin. Alors que se concentrent dans le bourg de Bréhat des sociétés à vocation commerciale (SARL, SA), les petits îlots environnants appartiennent en partie ou intégralement à des SCI (Béniguet, Logodec, etc.). Les propriétés appartenant à des SCI sont moins nombreuses sur l'île principale et leur localisation reste avant tout littorale (cf. les grandes propriétés situées à Kerarguillis (île nord) ou autour de l'étang du Birlot [île sud], fig. 3).

Enfin, parmi les collectivités privées détentrices de foncier sur les deux îles, soulignons la présence ponctuelle mais circonscrite de biens détenus par l'Université Paris I (terrains sis auprès de la pointe du Paon [Bréhat]) ou par l'Aérospatiale ou l'auberge de jeunesse (pointe sud-est de Batz).

16 Les particuliers étant majoritaires, une cartographie spécifique apparait nécessaire pour appréhender la répartition des résidents et des non-résidents. Cette division, établie en 
fonction du lieu de domicile déclaré dans les matrices cadastrales, distingue donc les résidents permanents (ou par simplification les insulaires) et les résidents secondaires (domicile en dehors de l'île).

\section{L'organisation de l'espace : reflet de la propriété foncière?}

17 À eux seuls les résidents secondaires sont aujourd'hui propriétaires de près de $60 \% \mathrm{du}$ territoire communal bréhatin : ils détiennent ainsi près de 6 fois la surface possédée par les résidents permanents (tab. 1). Le poids de l'activité touristique, et à travers lui celui du phénomène de résidences secondaires, se traduit donc par une possession massive du sol par les habitants non permanents. À l'inverse, la propriété foncière à Batz est directement liée à la persistance de l'agriculture maraîchère : les Batziens possèdent près de $45 \%$ de la superficie de l'île et sur les 10 plus gros propriétaires terriens, 5 sont encore des habitants de Batz, certains détenant plus de 5 ha - alors qu'à Bréhat, on ne recense qu'un seul insulaire parmi les 10 plus gros propriétaires que compte la commune.

Comment se répartissent habitants permanents et secondaires au sein de ces deux espaces insulaires? Quelles sont les différences et comment les expliquer?

D'emblée, la figure 4 souligne une première distinction entre les deux îles : alors que les "particuliers domiciliés dans l'île » sont à la fois nombreux et se répartissent de façon homogène à Batz, leur présence est beaucoup plus réduite et diffuse à Bréhat.

20 À Batz, les particuliers résidents constituent les plus grands propriétaires fonciers, il est donc normal qu'on les identifie facilement. Sans être absents du bourg et de la côte sud de l'île, ils se concentrent davantage dans la partie centrale de l'île; zone privilégiée des cultures maraîchères pratiquées sur de petites parcelles (d'où le morcellement prononcé et observable sur la figure 3 et la photo 1). Cette partie de l'île finistérienne est d'ailleurs classée en zone $\mathrm{N}$ dans le plan cadre ${ }^{6}$. D'ailleurs les propriétaires non-résidents sont peu nombreux dans cette partie de l'île (fig. 4). Cantonnés, semble-t-il, au bourg, leur présence y est très ponctuelle, excepté pour les propriétaires domiciliés dans la région Bretagne; ce qui s'explique le plus souvent par des attaches familiales sur l'île, à l'origine d'un patrimoine important (ils détiennent près de $15 \%$ de la superficie de l'île). Les propriétaires domiciliés en Île-de-France sont encore moins nombreux, ils possèdent seulement $6 \%$ de l'île, consistant essentiellement en quelques propriétés localisées sur la côte sud. Quant aux autres résidents secondaires propriétaires, leur poids est faible et eux aussi se concentrent davantage dans le bourg et à la périphérie de la zone agricole. 
Photo 1 : Parcelles cultivées, Nord de Batz (2001)

Cultivated fields, North of Batz

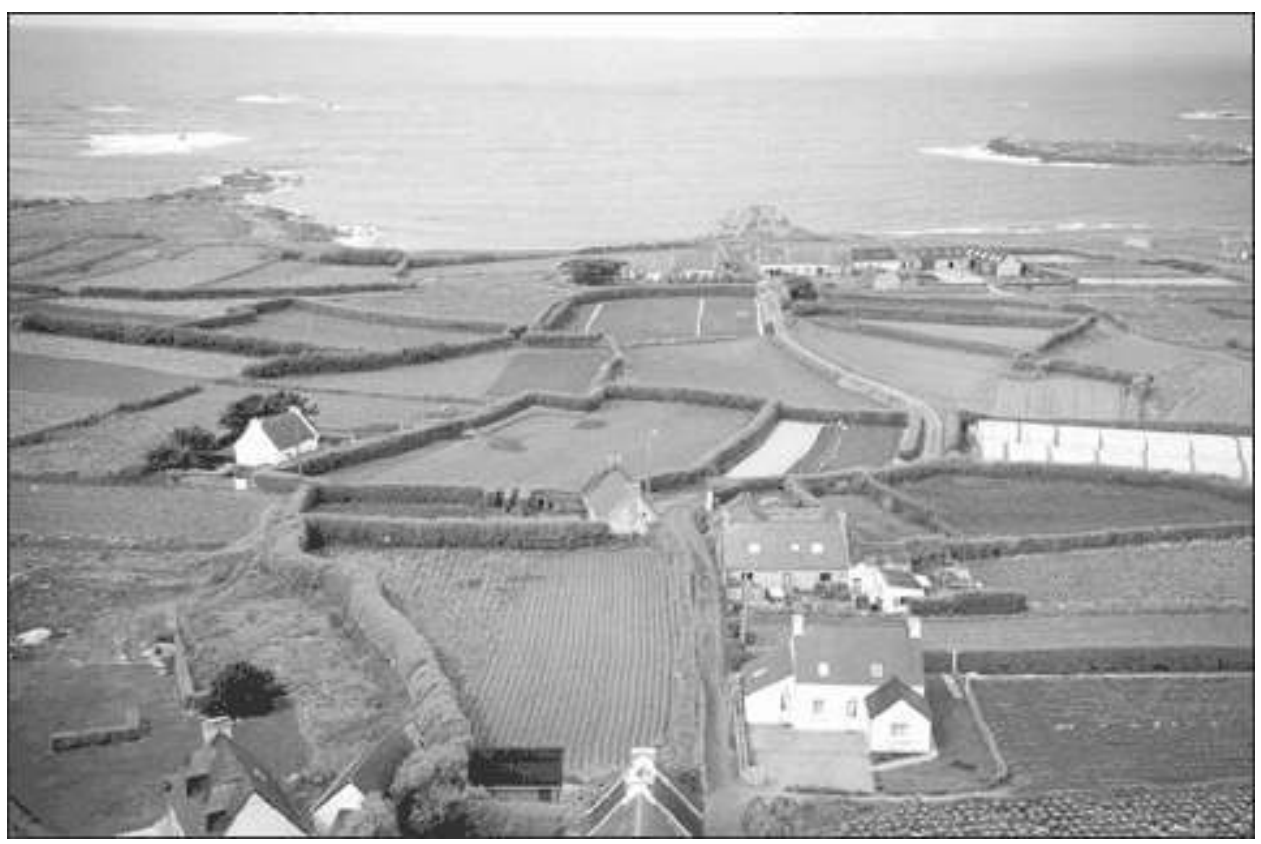

(cl. C. Buhot)

Figure 4 : répartition géographique des particuliers propriétaires à Bréhat et à Batz (2002) Geographical distribution of individual owners on Bréhat and Batz Islands (2002

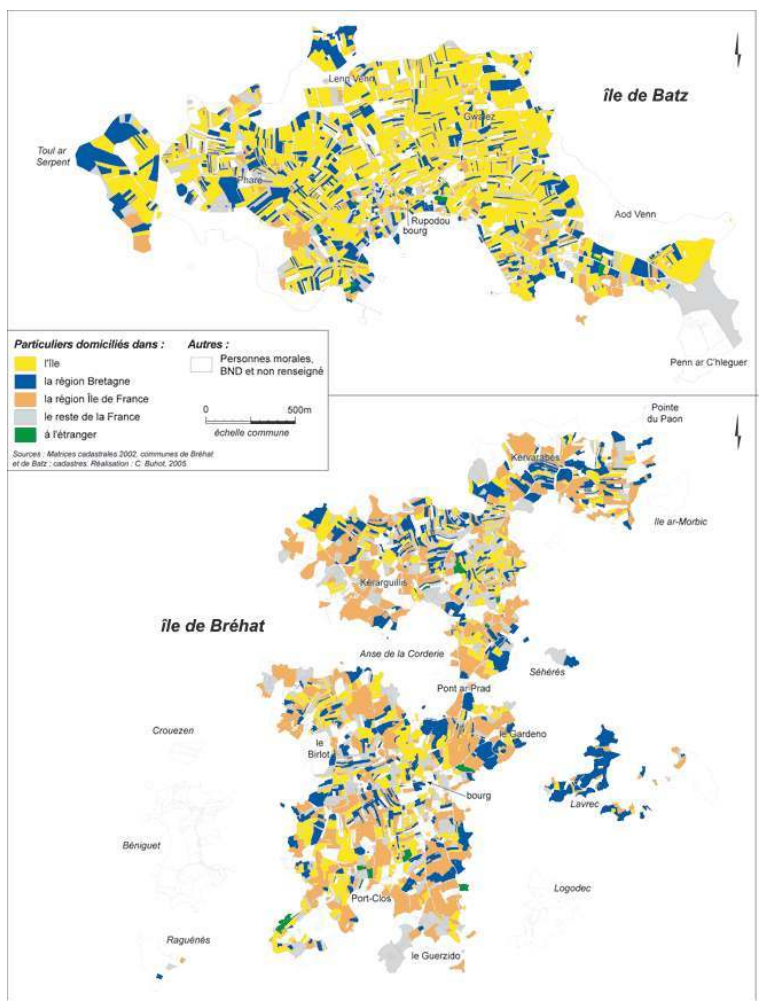

21 Représentant $44 \%$ de la surface totale de Batz (Brigand, 2002), l'agriculture freine visiblement l'acquisition et la détention de foncier des résidents secondaires. Mais plus 
que les terrains, ce qui intéresse avant tout ces derniers, c'est de posséder une maison: alors que moins de $30 \%$ de la superficie de l'île leur appartient, ils sont déjà propriétaires de plus de la moitié des logements.

Dans ce contexte, la localisation des propriétés appartenant aux non-résidents résulte de l'organisation structurelle de l'espace batzien : l'habitat étant essentiellement concentré à proximité du port et le long des axes de communication, c'est là que se concentre la majorité des résidences secondaires. Et l'agriculture, en monopolisant la totalité des terres exploitables, cantonne d'une part l'habitat sur des étendues limitées; d'autant plus que seuls le bourg et les secteurs du Phare et de Gwalez peuvent encore acquérir de nouvelles constructions; et d'autre part explique la rareté des propriétaires nonrésidents dans la partie centrale de l'île.

À Bréhat, les résidents ne sont plus les principaux propriétaires fonciers: ils ne détiennent d'ailleurs plus qu'un dixième de la superficie de l'île; d'où une plus grande difficulté à les repérer sur la figure 4. Principalement regroupés dans la partie ouest de l'île sud, les propriétaires résidents sont quasiment absents de secteurs comme celui du Guerzido (sud-est) et du Gardeno (sud de Pont ar Prat). Dans l'île nord, les propriétés sont la plupart du temps composées de parcelles morcelées et isolées; la seule présence notable des propriétaires résidents dans cette partie de l'île se résume à quelques îlots de propriété au nord du pont.

L'ascendant pris par les propriétaires non-résidents est remarquable à Bréhat: statistiquement comme nous l'avons évoqué et spatialement comme l'illustre la figure 4. Omniprésents tant sur l'île nord que sur l'île sud, les résidents secondaires possèdent en premier lieu les grandes propriétés littorales, en privilégiant quelques secteurs tout de même : le Guerzido, le Gardeno, le Birlot et l'ouest de l'île nord.

L'architecture (maisons de style ou propriétés en granit rose, cf. photo 2), la vue et le calme semblent autant d'éléments recherchés par les résidents secondaires. Et alors que $76 \%$ des logements sont déjà entre leurs mains, ils détiennent également $60 \%$ de la surface communale. L'acquisition des terrains jouxtant leur propriété est aisée et il arrive qu'ils détiennent aussi des parcelles fort distantes de celle-ci; le vendeur profite de la vente pour s'en dessaisir; ce qui explique la détention de nombreuses parcelles situées dans l'île nord sans lien avec la propriété bâtie. Les héritages successifs ont également contribué à morceler le parcellaire (physiquement et entre propriétaires). Les terrains en question, étant situés en zone protégée (site classé + loi littoral), sont sans aucune valeur marchande, leurs propriétaires n'ont donc pas jugé utile de procéder soit à un partage des parcelles entre héritiers soit à des remembrements ponctuels. 
Photo 2 : Concentration de résidences secondaires, Guerzido - Bréhat (2001) Concentration of second homes, Guerzido - Bréhat (2001)

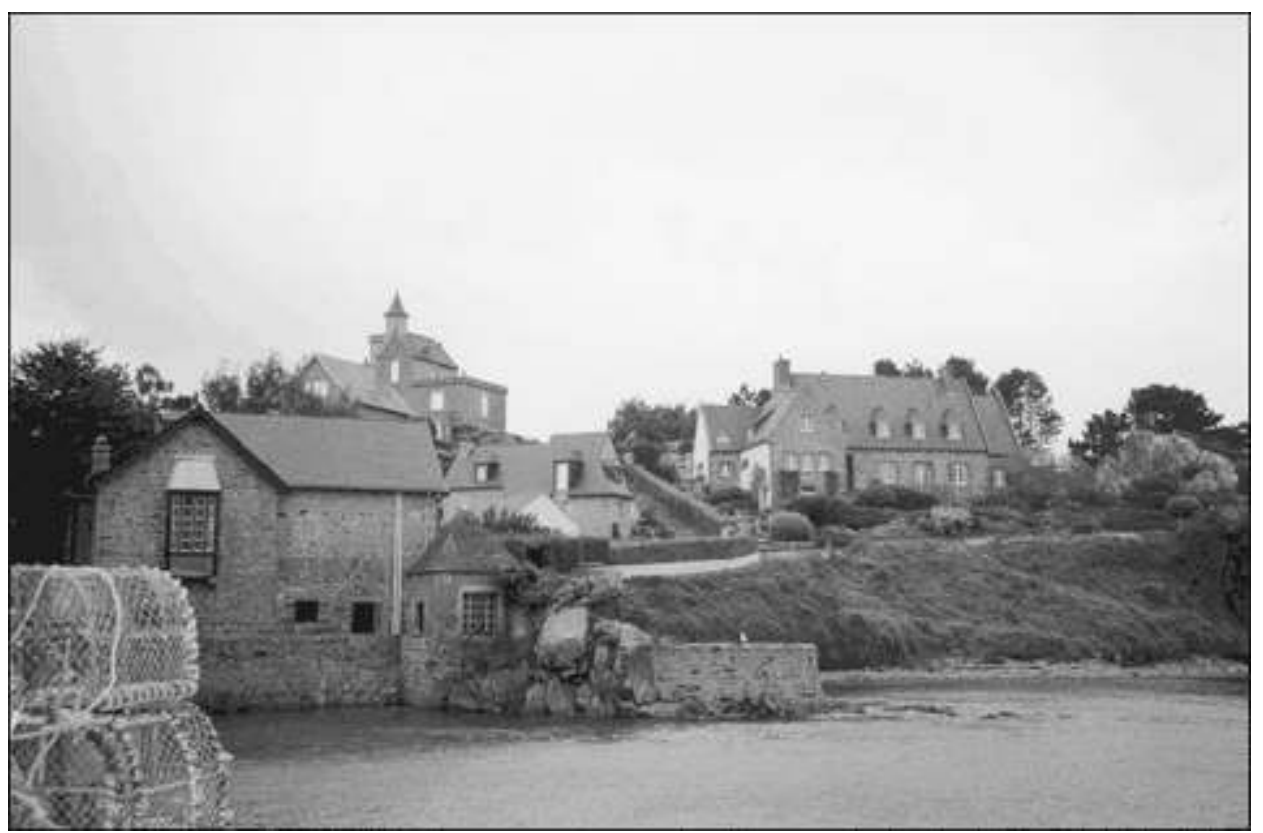

(cl. C. Buhot)

L'analyse de la propriété foncière, et à travers elle l'identification des différents types de
propriétaires, permet donc de comprendre les logiques de l'organisation d'un territoire
communal. À l'inverse de Bréhat où l'absence d'activité palliative au tourisme résidentiel

Ainsi, il résulte que pour la majorité des propriétaires domiciliés en dehors de l'île, la localisation est principalement littorale ; la taille des propriétés augmentant d'ailleurs du centre de l'île vers les côtes. Peut-être cette localisation privilégiée est-elle plus nette pour les particuliers résidant à l'étranger (mais ils ne sont pas nombreux) et ensuite pour ceux domiciliés en Bretagne (leur patrimoine foncier se réduit à de simples parcelles dans la partie centrale de l'île sud et nord de l'île nord). Quant aux propriétaires d'île de France et du reste du pays, notons encore une fois que l'on retrouve les plus grandes propriétés sur le bord de mer.

Cette répartition paraît alors ou bien dictée par la volonté de posséder une résidence secondaire bénéficiant d'une vue sur mer ou bien de s'éloigner du centre de la vie insulaire que constitue le bourg et sa périphérie. Mais peut-être les deux raisons sontelles complémentaires.

Résultant de la propriété foncière énoncée plus haut, l'organisation de l'espace se décompose ainsi : une zone centrale : bourg et sa périphérie + partie intérieure de l'île, où les propriétaires non résidents sont peu présents, et une zone côtière où ils sont surreprésentés. C'est donc l'habitat des résidents secondaires qui semble conditionner celui des insulaires; puisque ces derniers ne se concentrent pas dans un secteur particulier. Cette répartition entre propriétaires résidents et non-résidents traduit finalement la dispersion de l'habitat, constatée sur Bréhat.

\section{Conclusion et perspectives}


accélère l'acquisition de terres par des résidents secondaires, la propriété foncière à Batz dicte encore, à travers l'agriculture, l'organisation spatiale de l'île. logique plus opérationnelle. La collecte des matrices cadastrales ne constitue que la base d'un SIG où de nouvelles couches peuvent être créées et intégrées. Ces dernières pourraient inclure le plan d'urbanisme, l'occupation du sol, les mesures de protection, etc. Ce qui nous permettrait de définir un document de prospective pour la municipalité. « La variable foncière est une clé de bien des enjeux du littoral » précisait la DATAR (2004) dans son récent rapport intitulé Construire ensemble un développement équilibré du littoral, et d'ajouter qu'elle « a trop longtemps été négligée par les acteurs publics en charge de cet espace ».

31 La réalisation de ce type de travail montre que les données relatives à la question foncière sont facilement mobilisables et utilisables et que des applications concrètes pourraient voir le jour si elles étaient partagées par l'ensemble des acteurs. La DATAR (2004) signalait à ce propos la réticence de ces derniers à mettre en commun leurs données comme un motif d'échec aux projets d'observatoires locaux, départementaux ou régionaux.

Enfin, les données ainsi collectées nous incitent à évoquer d'autres pistes comme celle des usages : qui sont précisément les propriétaires, sont-ils originaires de l'île et sinon comment et pourquoi ont-ils choisi d'y acquérir un pied-à-terre? Quelles sont leurs pratiques des lieux? Notre analyse apporte déjà quelques réponses à ces questions, puisque nous avons remarqué que la possession des terres sur les îles reflète les zones préférentielles d'émigration des deux populations insulaires, mais elle nous amène à nous poser d'autres questions, dont les réponses passent vraisemblablement par des enquêtes de terrain. Comme l'affirmait déjà R. Trapitzine en 1982: «Les propriétaires sont les détenteurs du sol support et figurent de ce fait parmi les facteurs clefs de toute politique d'aménagement. La connaissance du comportement des propriétaires pour être appréhendée globalement ne peut s'effectuer qu'au travers d'une analyse des données foncières complétées sur le terrain par une approche socio-économique. »

\section{BIBLIOGRAPHIE}

BRIGAND (L.), 2002. - Les îles du Ponant. Histoires et géographie des îles et archipels de la Manche et de l'Atlantique, Plomelin, Palantines, $480 \mathrm{p}$.

BRIGAND (L.), LE DÉMÉZET (M.), FICHAUd (B.), 1986. - Les changements écologiques, économiques et sociologiques dans les îles du Ponant : le cas de Batz, Ouessant et Groix, Rapport Université de Bretagne Occidentale, Brest, $200 \mathrm{p}$.

Buнот (C.), 2004. - Analyse du marché foncier et immobilier dans les îles du Ponant (1995-2001), rapport Association des îles du Ponant, 53 p. [disponible sur le site www.iles-du-ponant.com/bases/pdf/ documentation/fr].

Chaussende (P.), 1994. - « Le parcellaire, témoin de l'histoire », Études Foncières, nº 62, p. 37-38. 
COMBY (J.), ReNARD (V.), 1996. - Les politiques foncières, Paris, PUF, coll. « Que sais-je ?», 126 p.

DALigauX (J.), 1996. - Structures foncières et processus d'urbanisation en milieu rural et périurbain. Le cas du massif des Maures (Var), Thèse de doctorat de Géographie humaine, Université d'Aix-

Marseille I, $337 \mathrm{p}$.

DATAR, 2004. - Construire ensemble un développement équilibré du littoral, Paris, La Documentation française, $156 \mathrm{p}$.

DE MoncAn (P.), 2002. - À qui appartient la France ?, Éditions du Mécène, 278 p.

TRAPITZINE (R.), 1982. - « Informatiser le foncier pour mieux gérer l'espace », Études Foncières, $\mathrm{n}^{\circ}$ 16 , p. 2-4.

\section{Sources}

Matrices cadastrales, 2002. - communes de Bréhat et de Batz.

Cadastres 2002. - Hôtels des Impôts de Morlaix et Saint-Brieuc.

\section{NOTES}

1. Les Centres des Impôts disposent de ces tableaux sous une forme numérique mais qu'il est impossible au public de consulter.

2. La distinction résidents permanents/résidents secondaires opérée ici s'appuie sur un critère statistique unique : le lieu de résidence déclaré dans les matrices cadastrales. Ainsi, est considéré comme résident secondaire toute personne non domiciliée dans l'île. Cette remarque est valable pour l'ensemble de l'article.

3. Groupement de personnes, représentant une collectivité d'intérêts, doté d'une personnalité juridique, distincte de celle de chacun des membres qui le composent.

4. L'objectif d'une SCI (Société Civile Immobilière) est de gérer et d'administrer plusieurs biens achetés ou reçus en apport. En règle générale, les SCI pérennes sont des $\mathrm{SCI}$ de famille dont les porteurs de part sont tous des parents et sont constituées en vue d'une simplification des problèmes de succession (De Moncan, 2002).

5. L'avantage est d'éviter les frais de notaire, les frais de publication aux hypothèques et un recours au géomètre, coûts inhérents à toute division de parcelle.

6. Document d'urbanisme de référence (datant de 1997). Il sera prochainement remplacé par un PLU.

\section{RÉSUMÉS}

L'étude de la structure de la propriété foncière participe à la compréhension de l'organisation spatiale du territoire. Mais comment identifier la propriété foncière et ses acteurs? Les matrices cadastrales et leur intégration dans un Système d'Information Géographique permettent de cartographier précisément les types de propriétaires et leur répartition au sein d'une commune. 
À partir du cas de deux îles, Bréhat et Batz, l'article entend établir un parallèle entre la propriété foncière (et notamment les types de propriétaires fonciers rencontrés) et l'organisation spatiale. Ainsi à Batz, les propriétaires résidents sont les principaux détenteurs de foncier. Leur concentration dans la partie centrale de l'île illustre la persistance de l'agriculture maraîchère. À l'inverse, à Bréhat, le sol appartient majoritairement à des propriétaires non-résidents. Cette situation résulte $\mathrm{du}$ poids de l'activité touristique, et particulièrement du phénomène des résidents secondaires ; car bien que surreprésentées sur le bord de mer, les propriétés des nonrésidents sont à l'image de l'habitat, dispersées sur le territoire communal.

Studying the framework of real estate helps understand the organisation of space. But how can we identify real property and those bearing on it? The cadastre together with integrating it into a geographic information system make it possible to draw precise maps of owners and the way they are dispatched in the district (or parish). Taking into account two islands, Bréhat and Batz, this article wants to draw a parallel between real property (more particularly the category of owners who have been met) and the organisation of space.

Thus on Batz island the locals are mainly the owners of real estate, they are concentrated in the middle part of the island which shows how market gardening persists there. Conversely on Bréhat island the real estate mainly belongs to non residents. It results from the importance of the tourist industry and more particularly from the development of secondary residences since, though they are overwhelmingly present on the coast, these non residents properties are typically scattered to the image of the rest of the island.

\section{INDEX}

Keywords : agriculture, cadastre, GIS, island, real property, second home residents Index géographique : Côtes-d'Armor, France, île de Batz, Îles de Bréhat

Mots-clés : agriculture, cadastre, île, propriété foncière, résidents secondaires, SIG

\section{AUTEUR}

\section{CLOTILDE BUHOT}

Université de Bretagne Occidentale (Brest), GEomer, UMR 6554 LETG - CNRS,

Clotilde.Buhot@univ-brest.fr 